\title{
An Investigation of Thermally Induced Collagen Structural Transitions by Second Harmonic Generation Microscopy
}

Yen Sun ${ }^{1}$, Sun-Jan Lin ${ }^{2,3}$, Chih-Yuan Hsiao, Wen Lo ${ }^{1}$, Shiou-Hwa Jee ${ }^{2,3}$, Chen-Yuan Dong ${ }^{1 *}$.

'Department of Physics, National Taiwan University, Taipei, Taiwan Republic of China, ${ }^{2}$ College of Medicine, National Taiwan University, Taipei, Taiwan Republic of China, ${ }^{3} \mathrm{National}$ Taiwan University Hospital, Taipei, Taiwan Republic of China. ${ }^{4}$ Institute of Electro Optics, Department of Electrical Engineering, National Taiwan University, Taipei 100, Taiwan

*Corresponding author: Chen-Yuan Dong

Tel: 3366-5155 Fax:3366-5244 E-mail; cydong@phys.ntu.edu.tw

Abstract In this work, the thermal disruption of type I collagen is investigated using second harmonic generation (SHG) microscopy. We investigated the effects of second harmonic generation images and intensity of rat tail tendon in the temperature range from 25 to $60^{\circ} \mathrm{C}$. We found a structural transition at $57^{\circ} \mathrm{C}$ where a drop in second harmonic intensity is correlated to a disorganization of the collagen fibers. Our results suggest that SHG microscopy is an effective technique to monitor thermally induced collagen structural changes for biomedical applications.

Methods and Results In recent years, multiphoton fluorescence microscopy has gained significant popularity in bioimaging applications. The non-linear excitation of fluorescence photons using ultrafast, near-infrared excitation sources have important advantages in its ability to acquire enhanced axial discrimination images, reduced overall specimen photodamage, and increased imaging penetration depths. In biological structures lacking an inversion symmetry, a non-vanishing second order susceptibility can contribute to a second harmonic generation (SHG) signal given by

$$
P_{i}=\chi_{i j k}^{2} E_{j} E_{k}
$$

The collagen is widely found in tissues such as the tendon, skin, and the cornea and is a major constituent of the extracellular matrix. A particularly interesting application of SHG imaging of collagen is the thermally induced structural transitions of collagen fibers. A number of medical procedures depend on heat induced changes in collagen fibers to achieve therapeutic results. In laser assisted capsulorrhaphy, laser heating of the shoulder collagen can result in fiber shrinkage and results in enhanced stability of the shoulder joint.

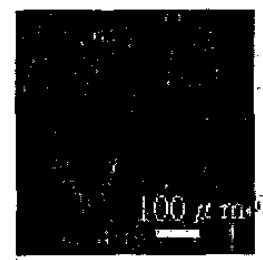

25

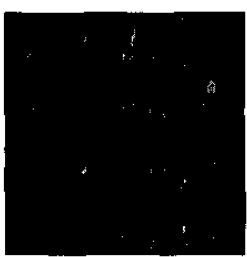

55

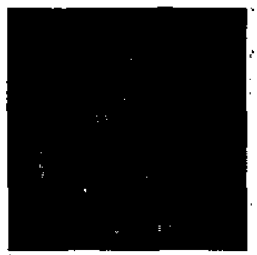

57

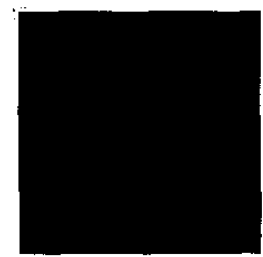

60

Figure 1 Second harmonic generation images of rat tail tendon treated for one hour at the temperature indicated. 


\section{THP-25(T5)}

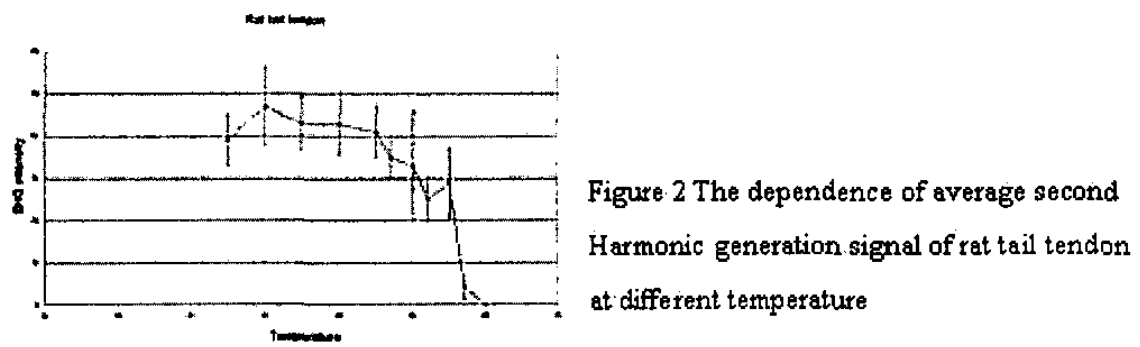

In this work, we obtained the SHG images from rat tail tendon after thermal treatment at the temperature range between 25 and $60^{\circ} \mathrm{C}$, at 5 degrees steps. We correlate the structural changes of collagen to changes to its SHG signal. From Fig 1 we found out that the structure of the collagen start to become discontinuous at $57^{\circ} \mathrm{C}$, before the SHG intensity drop to zero, and at $60^{\circ} \mathrm{C}$, the structure has been completely disrupted. Fig. 2 shows that the changes in SHG signal as a function of temperature. We found out there is a sudden drop between $55^{\circ} \mathrm{C}$, to $60^{\circ} \mathrm{C}$. By comparing Fig 1 with Fig 2 we can see that the sudden drop of SHG intensity is most likely due to the structure change in the rat tail tendon.

Conclusion In conclusion, SHG microscopy of rat tail tendon treated for ten min at different temperatures show that collagen I undergoes a structural transition at $57 \mathrm{C}$. where a change in the microscopic structure of collagen fibers results in a disruption in the inversion-symmetry lacking structure that produces the SHG signal. We suggest that from the SHG imaging of collagen we can investigate the changes in its structure due to thermal effect. Our investigation will help better understand the thermal effect of collagen structure and devise more effective strategies in heat-assisted therapeutic procedures of collagen.

\section{Reference}

1. W. Denk, J. H. Strickler, and W. W. Webb, Science 248(4951), 73 (1990).

2. P. T. C. So, C. Y. Dong, B. R. Masters, and K. M. Berland, Annual Review of Biomedical Engineering 2, 399 (2000).

3. P. J. Campagnola and L. M. Loew, Nature Biotechnology 21(11), 1356-1360 (2003).

4. A. Zoumi, A. Yeh, and B. J. Tromberg, Proceedings of the National Academy of Sciences of the United States of America 99(17), 11014-11019 (2002).

5. T. Theodossiou, G. S. Rapti, V. Hovhannisyan, E. Georgiou, K. Politopoulos, and D. Yova, Lasers in Medical Science 17(1), 34-41 (2002).

6. B. M. Kim, J. Eichler, K. M. Reiser, A. M. Rubenchik, and L. B. Da Silva, Lasers in Surgery and Medicine 27(4), 329-335 (2000).

7. T. R. Lyons, P. L. Griffith, F. H. Savoie, and L. D. Field, The Joumal of Arthroscopic and Related Surgery, 17(1):25-30 (2001).

8. M. B. McDonald, P. S. Hersh, E. E. Manche, R. K. Maloney, J. Davidorf, M. Sabry, and the Conductive Keratoplasty United States Investigators Group, Ophthalmology, 109:1978-1989 (2002).

9. R. E. Fitzpatrick, M. P. Goldman, N. M. Satur, and W. D. Tope, Archives of Dermatology, 132(4):395-402 (1996).

10. Y. Sun, J. W. Su, W. Lo, S. J. Lin, S. H. Jee, and C. Y. Dong, Optics Express, 11(25): 3377 (2003). 